\title{
Equation-of-Motion Coupled-Cluster Protocol Calculating Magnetic Properties: Theory and Applications to Single-Molecule Magnets
}

\author{
Maristella Alessio and Anna I. Krylov \\ Department of Chemistry, University of Southern California, Los Angeles, California 90089-0482, USA
}




\section{Matrix representations of orbital momentum opera- tors in the basis of spherical harmonics ( $d$ block)}

This section gives matrix representations of the orbital angular momentum operators (taken from Ref. 1) in the basis of real harmonics, $Y_{L, M_{L}}$, where $L$ specifies the orbital momentum and $M_{L}$ is its projection on the z-axis. In the case of $L=2$, we have $Y_{2,-2}=d_{x y}, Y_{2,-1}=d_{y z}$, $Y_{2,0}=d_{z^{2}}, Y_{2,+1}=d_{x z}$, and $Y_{2,2}=d_{x^{2}-y^{2}}$. The corresponding matrix elements explain ElSayed's rules for the manifold of $d$-orbitals. ${ }^{1}$

$$
\begin{aligned}
& \left|Y_{2,-2}\right\rangle \quad\left|Y_{2,-1}\right\rangle \quad\left|Y_{2,0}\right\rangle \quad\left|Y_{2,1}\right\rangle \quad\left|Y_{2,2}\right\rangle \\
& \begin{array}{l}
\left\langle Y_{2,-2}\right| \\
\left\langle Y_{2,-1}\right| \\
\left\langle Y_{2,0}\right| \\
\left\langle Y_{2,1}\right| \\
\left\langle Y_{2,2}\right|
\end{array}\left(\begin{array}{ccccc}
0 & 0 & 0 & 0 & 2 i \\
0 & 0 & 0 & i & 0 \\
0 & 0 & 0 & 0 & 0 \\
0 & -i & 0 & 0 & 0 \\
-2 i & 0 & 0 & 0 & 0
\end{array}\right) \\
& \left|Y_{2,-2}\right\rangle \quad\left|Y_{2,-1}\right\rangle \quad\left|Y_{2,0}\right\rangle \quad\left|Y_{2,1}\right\rangle \quad\left|Y_{2,2}\right\rangle
\end{aligned}
$$

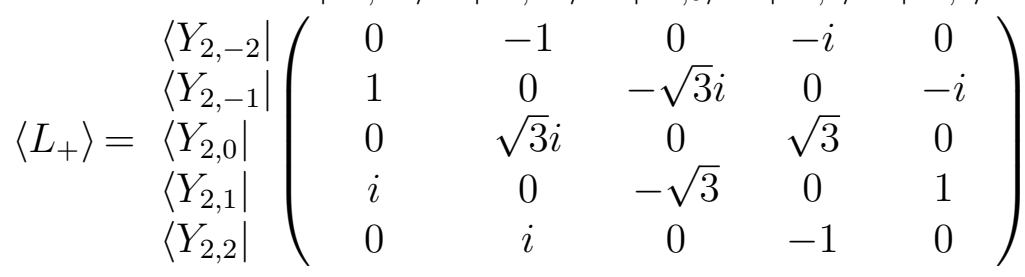

$$
\begin{aligned}
& \left|Y_{2,-2}\right\rangle \quad\left|Y_{2,-1}\right\rangle \quad\left|Y_{2,0}\right\rangle \quad\left|Y_{2,1}\right\rangle \quad\left|Y_{2,2}\right\rangle \\
& \left\langle Y_{2,-2}\right|\left(\begin{array}{ccccc}
0 & 1 & 0 & -i & 0 \\
-1 & 0 & -\sqrt{3} . & 0 & -i
\end{array}\right. \\
& \left\langle\begin{array}{cccccc}
\left\langle Y_{2,-1}\right| & -1 & 0 & -\sqrt{3} i & 0 & -i
\end{array}\right. \\
& \left\langle L_{-}\right\rangle=\left\langle Y_{2,0}\right| \quad \begin{array}{ccccc}
0 & \sqrt{3} i & 0 & -\sqrt{3} & 0
\end{array} \\
& \begin{array}{c}
\left\langle Y_{2,1}\right| \\
\left\langle Y_{2,2}\right|
\end{array}\left(\begin{array}{ccccc}
i & 0 & \sqrt{3} & 0 & -1 \\
0 & i & 0 & 1 & 0
\end{array}\right. \\
& \left|Y_{2,-2}\right\rangle \quad\left|Y_{2,-1}\right\rangle \quad\left|Y_{2,0}\right\rangle \quad\left|Y_{2,1}\right\rangle \quad\left|Y_{2,2}\right\rangle \\
& \left\langle Y_{2,-2}\right|\left(\begin{array}{ccccc}
0 & 0 & 0 & -i & 0
\end{array}\right) \\
& \left\langle Y_{2,-1}\right| \quad \begin{array}{lllll}
0 & 0 & -\sqrt{3} i & 0 & -i
\end{array} \\
& \left\langle L_{x}\right\rangle=\left\langle Y_{2,0}\right| \quad \begin{array}{lllll}
0 & \sqrt{3} i & 0 & 0 & 0
\end{array} \\
& \left\langle Y_{2,1}\right| \quad \begin{array}{lllll}
i & 0 & 0 & 0 & 0
\end{array} \\
& \left\langle Y_{2,2}\right|\left(\begin{array}{lllll}
0 & i & 0 & 0 & 0
\end{array}\right) \\
& \left|Y_{2,-2}\right\rangle \quad\left|Y_{2,-1}\right\rangle \quad\left|Y_{2,0}\right\rangle \quad\left|Y_{2,1}\right\rangle \quad\left|Y_{2,2}\right\rangle \\
& \begin{array}{c}
\left\langle Y_{2,-2}\right| \\
\left\langle Y_{2,-1}\right| \\
\left\langle Y_{2,0}\right| \\
\left\langle Y_{2,1}\right| \\
\left\langle Y_{2,2}\right|
\end{array}\left(\begin{array}{ccccc}
0 & i & 0 & 0 & 0 \\
-i & 0 & 0 & 0 & 0 \\
0 & 0 & 0 & -\sqrt{3} i & 0 \\
0 & 0 & \sqrt{3} i & 0 & -i \\
0 & 0 & 0 & i & 0
\end{array}\right)
\end{aligned}
$$




\section{Orbital angular momentum and spin-orbit matrix el- ements}

Table S1: Leading hole-particle NTO pair, orbital angular momentum and spin-orbit meanfield reduced matrix elements for the transition between states 1 and 2 of the trigonal pyramidal $[(\mathrm{tpa}) \mathrm{Fe}(\mathrm{II})]^{-}$, linear $\mathrm{Fe}(\mathrm{II})\left[\mathrm{C}\left(\mathrm{SiMe}_{3}\right)_{3}\right]_{2}$, and trigonal bipyramidal $\left(\mathrm{PMe}_{3}\right)_{2} \mathrm{Fe}(\mathrm{III}) \mathrm{Cl}_{3} \mathrm{com}-$ plexes. Only 1 (ket) $\rightarrow 2$ (bra) transition is shown. Effective energy barriers $U_{\text {eff }}$ are in $\mathrm{cm}^{-1}$ and are derived from ac susceptibility experiments.

\begin{tabular}{|c|c|}
\hline Fe(II) - Trigonal pyramidal & 1 (ket) $\rightarrow 2$ (bra) \\
\hline Leading hole-particle NTO pair & $\mathrm{d}_{y z} \rightarrow \mathrm{d}_{x z}$ \\
\hline$\left\langle Y_{L, M_{I}^{\prime}}\left|L_{z}\right| Y_{L, M_{L}}\right\rangle^{a}$ & $\left\langle Y_{2,1}\left|L_{z}\right| Y_{2,-1}\right\rangle=-i$ \\
\hline$\left\langle 2\left|L_{z}\right| 1\right\rangle^{b}$ & $0.99 i$ \\
\hline$\left\langle S\left|H_{L_{-}}^{\mathrm{SOMF}}\right| S\right\rangle$ & $-0.29+0.09 \mathrm{i}$ \\
\hline$\left\langle S\left|H_{L_{0}}^{\mathrm{SOMF}}\right| S\right\rangle$ & $-211.32 \mathrm{i}$ \\
\hline$\left\langle S\left|H_{L_{+}}^{\mathrm{SOMF}}\right| S\right\rangle$ & $-0.29-0.09 \mathrm{i}$ \\
\hline$U_{\mathrm{eff}^{c}}$ & $65(42)$ \\
\hline Fe(II) - Linear & 1 (ket) $\rightarrow 2$ (bra) \\
\hline Leading hole-particle NTO pair & $\mathrm{d}_{x^{2}-y^{2}} \rightarrow \mathrm{d}_{x y}$ \\
\hline$\left\langle Y_{L, M_{L}^{\prime}}\left|L_{z}\right| Y_{L, M_{L}}\right\rangle^{a}$ & $\left\langle Y_{2,-2}\left|L_{z}\right| Y_{2,2}\right\rangle=2 i$ \\
\hline$\left\langle 2\left|L_{z}\right| 1\right\rangle^{b}$ & $-1.95 i$ \\
\hline$\left\langle S\left|H_{L_{-}}^{\mathrm{SOMF}}\right| S\right\rangle$ & $-2.45-2.25 \mathrm{i}$ \\
\hline$\left\langle S\left|H_{L_{0}}^{\mathrm{SOMF}}\right| S\right\rangle$ & $422.76 \mathrm{i}$ \\
\hline$\left\langle S\left|H_{L_{+}}^{\mathrm{SOM}_{0}}\right| S\right\rangle$ & $-2.45+2.25 \mathrm{i}$ \\
\hline$U_{\mathrm{eff}^{d}}$ & 146 \\
\hline Fe(III) - Trigonal bipyramidal & 1 (ket) $\rightarrow 2$ (bra) \\
\hline Leading hole-particle NTO pair & $\mathrm{d}_{x z} \rightarrow \mathrm{d}_{y z}$ \\
\hline$\left\langle Y_{L, M_{L}^{\prime}}\left|L_{z}\right| Y_{L, M_{L}}\right\rangle^{a}$ & $\left\langle Y_{2,-1}\left|L_{z}\right| Y_{2,1}\right\rangle=i$ \\
\hline$\left\langle 2\left|L_{z}\right| 1\right\rangle^{b}$ & $-0.90 i$ \\
\hline$\left\langle S\left|H_{L_{-}}^{\mathrm{SOMF}}\right| S\right\rangle$ & $-2.75+0.07 i$ \\
\hline$\left\langle S\left|H_{L_{0}}^{\mathrm{SOMF}}\right| S\right\rangle$ & $218.75 \mathrm{i}$ \\
\hline$\left\langle S\left|H_{L_{+}}^{\mathrm{SOMF}}\right| S\right\rangle$ & $-2.75-0.07 i$ \\
\hline$U_{\mathrm{eff}^{e}}$ & 81 \\
\hline
\end{tabular}

${ }^{a}$ In the basis of real harmonics ( $d$-orbitals). ${ }^{b}$ By contracting the orbital-momentum integrals with the density matrix. ${ }^{c}$ For $\left[\left(\mathrm{tpa}^{\mathrm{Tbu}}\right) \mathrm{Fe}\right]^{-}\left(\left[\left(\mathrm{tpa}^{\mathrm{Mes}}\right) \mathrm{Fe}\right]^{-}\right)$from Ref. 2 (Ref. 2,3). ${ }^{d}$ Ref. 4.

e Ref. 5. 
Here, we also illustrate how to obtain $M_{L}$ by diagonalization of the matrix representation of $L_{z}$. From the $L_{z}$ matrix (computed between states 1 and 2 ) of $[(\mathrm{tpa}) \mathrm{Fe}(\mathrm{II})]^{-}$:

$$
\left\langle L_{z}\right\rangle=\begin{array}{cc}
|1\rangle & |2\rangle \\
\langle 2| & \langle 1|
\end{array}\left(\begin{array}{cc}
-0.26 i & -0.99 i \\
0.99 i & 0.31 i
\end{array}\right)
$$

we obtain eigenvalues

$$
M_{L}=\left(\begin{array}{cc}
0.95 & 0.00 \\
0.00 & -0.95
\end{array}\right)
$$

and the corresponding eigenvectors

$$
v=\left(\begin{array}{cc}
0.71 & -0.20+0.68 i \\
-0.20+0.68 i & 0.71
\end{array}\right)
$$

Similarly, for $\left(\mathrm{PMe}_{3}\right)_{2} \mathrm{Fe}(\mathrm{III}) \mathrm{Cl}_{3}$, we compute:

$$
\begin{gathered}
\left\langle L_{z}\right\rangle=\stackrel{\langle 1|}{\langle 2|}\left(\begin{array}{cc}
0.00 i & 0.90 i \\
-0.90 i & 0.00 i
\end{array}\right) \\
M_{L}=\left(\begin{array}{cc}
0.90 & 0.00 \\
0.00-0.90
\end{array}\right) \\
v=\left(\begin{array}{cc}
0.71 i & -0.71 i \\
0.71 & 0.71
\end{array}\right)
\end{gathered}
$$

These results are consistent with the ones derived by accounting for a larger number of multiplets (from 2 to 5 ). 


\section{Wave function analysis}

Table S2: Wave function properties of the five lowest eigenstates of the trigonal pyramidal $[(\mathrm{tpa}) \mathrm{Fe}(\mathrm{II})]^{-}$, linear $\mathrm{Fe}(\mathrm{II})\left[\mathrm{C}\left(\mathrm{SiMe}_{3}\right)_{3}\right]_{2}$, and trigonal bipyramidal $\left(\mathrm{PMe}_{3}\right)_{2} \mathrm{Fe}(\mathrm{III}) \mathrm{Cl}_{3}$ complexes. Energies are in $\mathrm{cm}^{-1}$. Effective numbers of unpaired electrons $\left(n_{u}\right.$ and $\left.n_{u, n l}\right)$ are computed using Head-Gordon's formulas. ${ }^{6}$

\begin{tabular}{lcccc}
\hline Fe(II) - Trigonal pyramidal & $E$ & $n_{u}$ & $n_{u, n l}$ & $\left\langle S^{2}\right\rangle$ \\
\hline$|1\rangle$ & 0 & 3.83 & 4.04 & 6.03 \\
$|2\rangle$ & 9 & 3.86 & 4.04 & 6.03 \\
$|3\rangle$ & 3715 & 4.19 & 4.07 & 6.11 \\
$|4\rangle$ & 9605 & 4.03 & 4.04 & 6.05 \\
$|5\rangle$ & 9683 & 4.03 & 4.03 & 6.05 \\
\hline Fe(II) - Linear & $E$ & $n_{u}$ & $n_{u, n l}$ & $\left\langle S^{2}\right\rangle$ \\
\hline$|1\rangle$ & 0 & 3.99 & 4.00 & 6.03 \\
$|2\rangle$ & 195 & 3.99 & 4.00 & 6.03 \\
$|3\rangle$ & 1689 & 4.11 & 4.02 & 6.06 \\
$|4\rangle$ & 5170 & 4.03 & 4.00 & 6.03 \\
$|5\rangle$ & 5228 & 4.03 & 4.00 & 6.03 \\
\hline Fe(III) - Trigonal bipyramidal & $E$ & $n_{u}$ & $n_{u, n l}$ & $\left\langle S^{2}\right\rangle$ \\
\hline$|1\rangle$ & 0 & 2.92 & 3.03 & 3.81 \\
$|2\rangle$ & 92 & 2.92 & 3.03 & 3.81 \\
$|3\rangle$ & 2074 & 4.68 & 5.01 & 8.73 \\
$|4\rangle$ & 7245 & 3.02 & 3.03 & 3.83 \\
$|5\rangle$ & 7447 & 3.04 & 3.04 & 3.83 \\
\hline
\end{tabular}




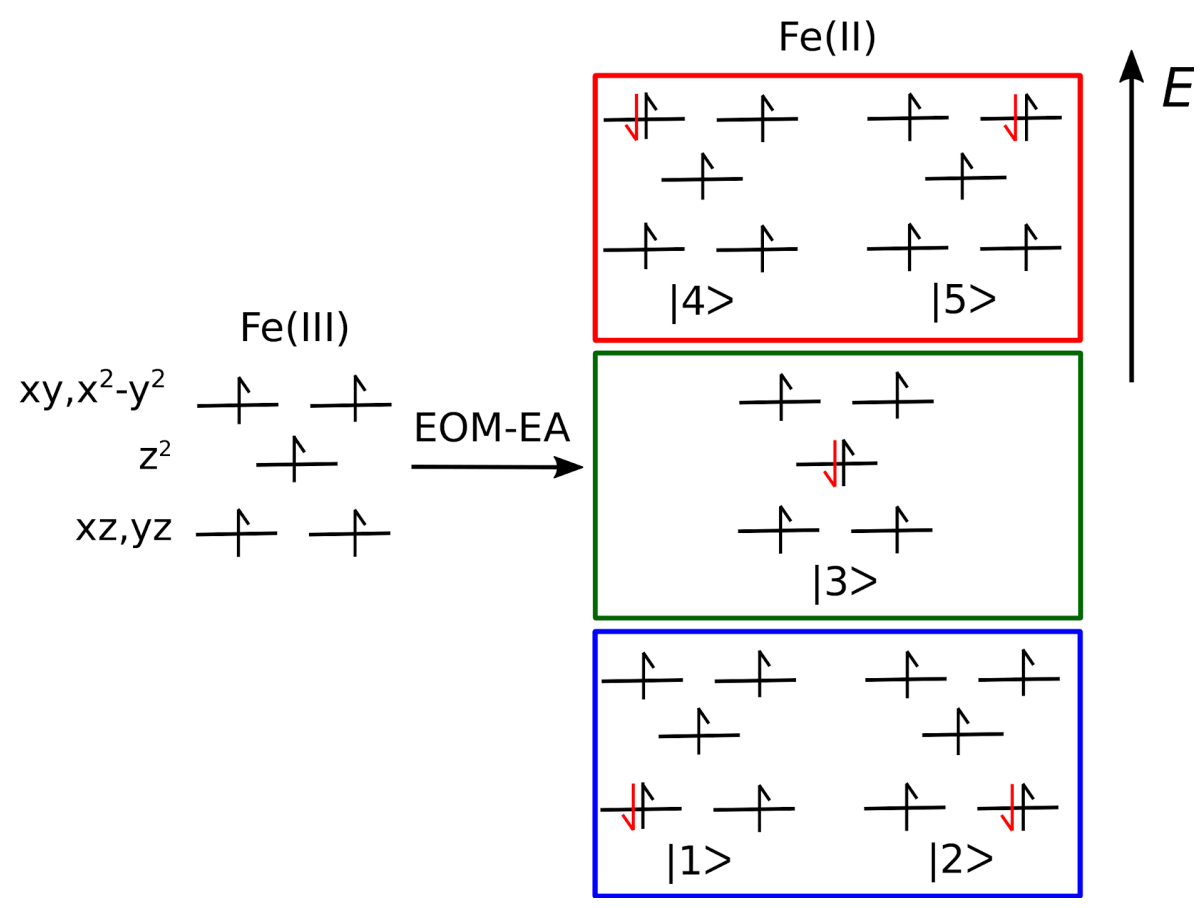

Figure S1: Electronic configurations of the reference hextet state of [(tpa)Fe(III)] and ground and excited quintet states of $[(\mathrm{tpa}) \mathrm{Fe}(\mathrm{II})]^{-}$. Target states (quintet $d^{6}$ configurations of $\mathrm{Fe}^{2+}$ ) are obtained by attachment of a $\beta$-electron (in red) to one of the singly occupied $d$-orbitals of the reference state (hextet $d^{5}$ configuration of $\mathrm{Fe}^{3+}$ ). 


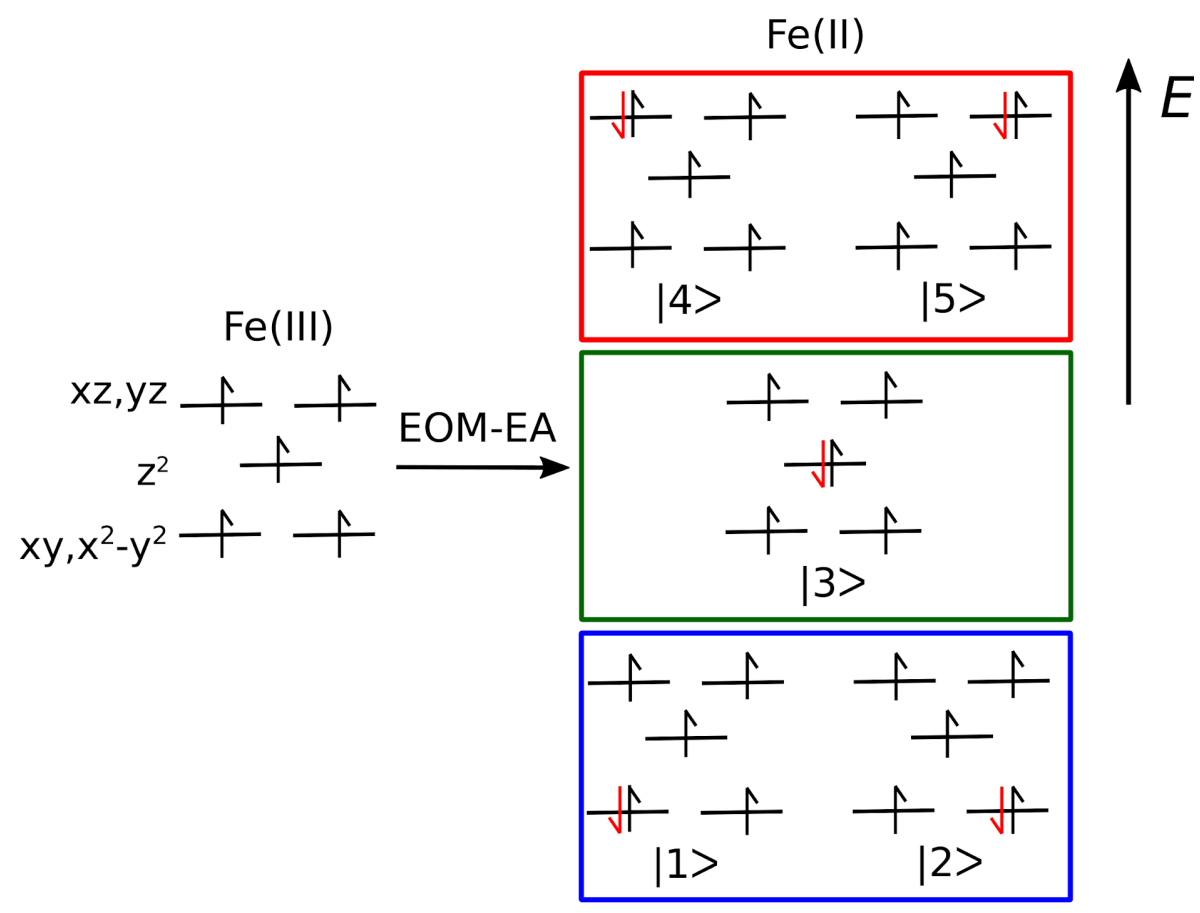

Figure S2: Electronic configurations of the reference hextet state of $\mathrm{Fe}(\mathrm{III})\left[\mathrm{C}\left(\mathrm{SiMe}_{3}\right)_{3}\right]_{2}^{+}$and ground and excited quintet states of $\mathrm{Fe}(\mathrm{II})\left[\mathrm{C}\left(\mathrm{SiMe}_{3}\right)_{3}\right]_{2}$. Target states (quintet $d^{6}$ configurations of $\mathrm{Fe}^{2+}$ ) are obtained by attachment of a $\beta$-electron (in red) to one of the singly occupied $d$ orbitals of the reference state (hextet $d^{5}$ configuration of $\mathrm{Fe}^{3+}$ ).

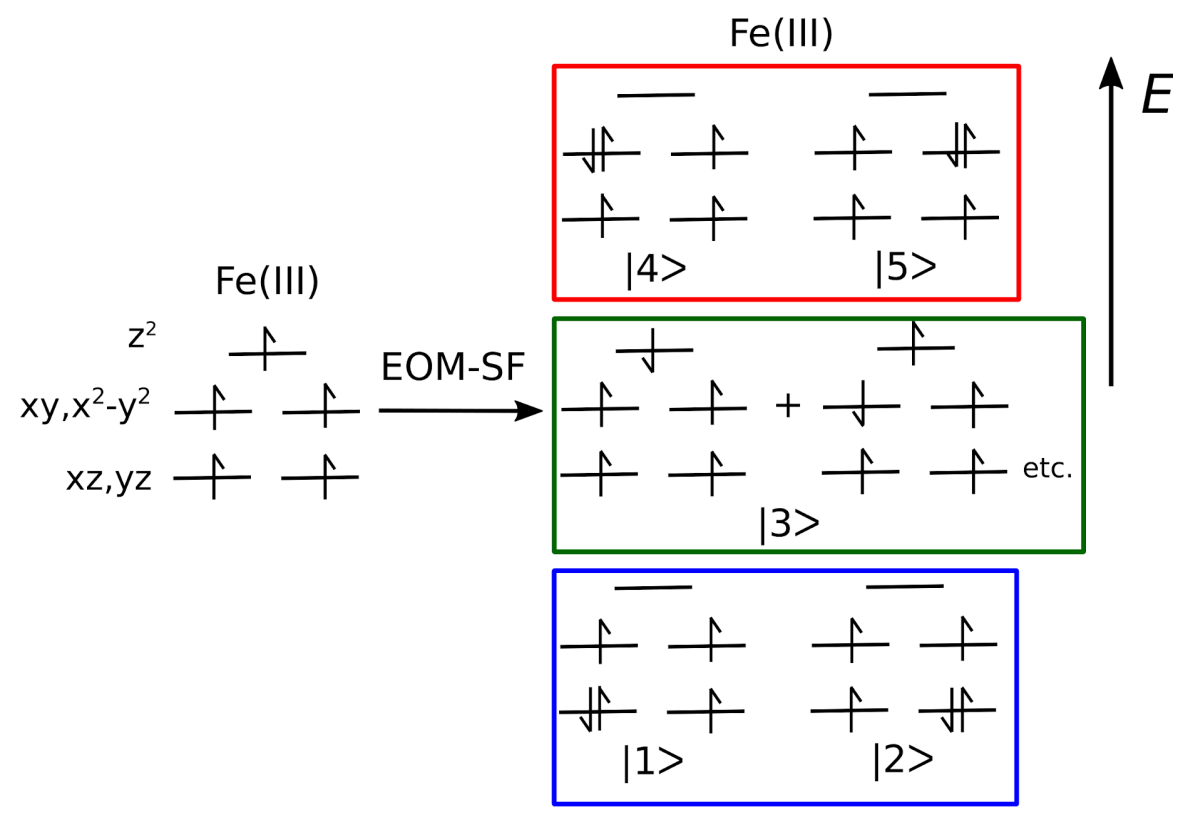

Figure S3: Electronic configurations of the high-spin hextet reference state and ground and excited states of $\left(\mathrm{PMe}_{3}\right)_{2} \mathrm{Fe}(\mathrm{III}) \mathrm{Cl}_{3}$. Target low-spin states (quartet and hextet $d^{5}$ configurations of $\mathrm{Fe}^{3+}$ ) are obtained by single spin-flip excitations from the high-spin hextet reference state of $\mathrm{Fe}^{3+}$. 


\section{Spin-orbit and Zeeman splitting}

Table S3: SOC- and field-corrected sublevels of the trigonal pyramidal $[(\text { tpa }) \mathrm{Fe}(\mathrm{II})]^{-}$, linear $\mathrm{Fe}(\mathrm{II})\left[\mathrm{C}\left(\mathrm{SiMe}_{3}\right)_{3}\right]_{2}$, and trigonal bipyramidal $\left(\mathrm{PMe}_{3}\right)_{2} \mathrm{Fe}(\mathrm{III}) \mathrm{Cl}_{3}$ complexes. SOC mixes the $2 S+1$ components of states $|1\rangle$ and $|2\rangle$, giving rise to $2(2 S+1)$ SOC-corrected sublevels that are two by two degenerate. Zeeman interactions lift this two-fold degeneracy. Energies are in $\mathrm{cm}^{-1}$. Field $\mathbf{H}$ along $z$-axis of $1 \mathrm{~T}$ (Tesla).

\begin{tabular}{|c|c|c|}
\hline Fe(II) - Trigonal pyramidal & $\mathrm{SOC}$ & SOC-Zee \\
\hline $\mid$ SOC-/SOC-Zee- $\left.1_{M_{S}=-2}\right\rangle$ & -173 & -175 \\
\hline $\mid$ SOC- $/$ SOC-Zee- $\left.2_{M_{S}=+2}\right\rangle$ & -173 & -170 \\
\hline$\left|\mathrm{SOC}-/ \mathrm{SOC}-\mathrm{Zee}-3_{M_{S}=-1}\right\rangle$ & -86 & -88 \\
\hline$\left|\mathrm{SOC}-/ \mathrm{SOC}-\mathrm{Zee}-4_{M_{S}=+1}\right\rangle$ & -86 & -85 \\
\hline$\left|\mathrm{SOC}-/ \mathrm{SOC}-\mathrm{Zee}-5_{M_{S}=0}\right\rangle$ & 0 & 0 \\
\hline$\left|\mathrm{SOC}-/ \mathrm{SOC}-\mathrm{Zee}-6_{M_{S}=0}\right\rangle$ & 0 & 0 \\
\hline$\left|\mathrm{SOC}-/ \mathrm{SOC}-\mathrm{Zee}-7_{M_{S}=+1}\right\rangle$ & 86 & 87 \\
\hline $\mid$ SOC- $/$ SOC $\left.-Z e e-8_{M_{S}=-1}\right\rangle$ & 86 & 86 \\
\hline$\left|\mathrm{SOC}-/ \mathrm{SOC}-\mathrm{Zee}-9_{M_{S}=+2}\right\rangle$ & 173 & 174 \\
\hline $\mid$ SOC- $/$ SOC-Zee- $\left.10_{M_{S}=-2}\right\rangle$ & 173 & 171 \\
\hline $\mathrm{Fe}(\mathrm{II})$ - Linear & $\mathrm{SOC}$ & SOC-Zee \\
\hline$\left|\mathrm{SOC}-/ \mathrm{SOC}-\mathrm{Zee}-1_{M_{S}=-2}\right\rangle$ & -261 & -264 \\
\hline $\mid$ SOC- $/$ SOC-Zee- $\left.2_{M_{S}=+2}\right\rangle$ & -261 & -258 \\
\hline $\mid$ SOC- $/$ SOC-Zee- $\left.3_{M_{S}=-1}\right\rangle$ & -101 & -102 \\
\hline $\mid$ SOC- $/$ SOC-Zee- $\left.4_{M_{S}=+1}\right\rangle$ & -101 & -99 \\
\hline$\left|\mathrm{SOC}-/ \mathrm{SOC}-\mathrm{Zee}-5_{M_{S}=0}\right\rangle$ & 0 & 0 \\
\hline$\left|\mathrm{SOC}-/ \mathrm{SOC}-\mathrm{Zee}-6_{M_{S}=0}\right\rangle$ & 195 & 195 \\
\hline $\mid$ SOC- $/$ SOC-Zee- $\left.7_{M_{S}=+1}\right\rangle$ & 296 & 296 \\
\hline$\left|\mathrm{SOC}-/ \mathrm{SOC}-\mathrm{Zee}-8_{M_{S}=-1}\right\rangle$ & 296 & 296 \\
\hline$\left|\mathrm{SOC}-/ \mathrm{SOC}-\mathrm{Zee}-9_{M_{S}=+2}\right\rangle$ & 456 & 457 \\
\hline $\mid \mathrm{SOC}-/ \mathrm{SOC}-\mathrm{Zee}-10_{\left.M_{S}=-2\right\rangle}$ & 456 & 455 \\
\hline Fe(III) - Trigonal bipyramidal & $\mathrm{SOC}$ & SOC-Zee \\
\hline$\left|\mathrm{SOC}-/ \mathrm{SOC}-\mathrm{Zee}-1_{M_{S}=-3 / 2}\right\rangle$ & -130 & -131 \\
\hline$\left|\mathrm{SOC}-/ \mathrm{SOC}-\mathrm{Zee}-2_{M_{S}=+3 / 2}\right\rangle$ & -130 & -128 \\
\hline$\left|\mathrm{SOC}-/ \mathrm{SOC}-\mathrm{Zee}-3_{M_{S}=-1 / 2}\right\rangle$ & -27 & -28 \\
\hline$\left|\mathrm{SOC}-/ \mathrm{SOC}-\mathrm{Zee}-4_{M_{S}=+1 / 2}\right\rangle$ & -27 & -26 \\
\hline$\left|\mathrm{SOC}-/ \mathrm{SOC}-\mathrm{Zee}-5_{M_{S}=+1 / 2}\right\rangle$ & 119 & 119 \\
\hline$\left|\mathrm{SOC}-/ \mathrm{SOC}-\mathrm{Zee}-6_{M_{S}=-1 / 2}\right\rangle$ & 119 & 119 \\
\hline$\left|\mathrm{SOC}-/ \mathrm{SOC}-\mathrm{Zee}-7_{M_{S}=+3 / 2}\right\rangle$ & 221 & 222 \\
\hline$\left|\mathrm{SOC}-/ \mathrm{SOC}-\mathrm{Zee}-8_{M_{S}=-3 / 2}\right\rangle$ & 221 & 220 \\
\hline
\end{tabular}




\section{$5 \quad$ Variable-field magnetization data}
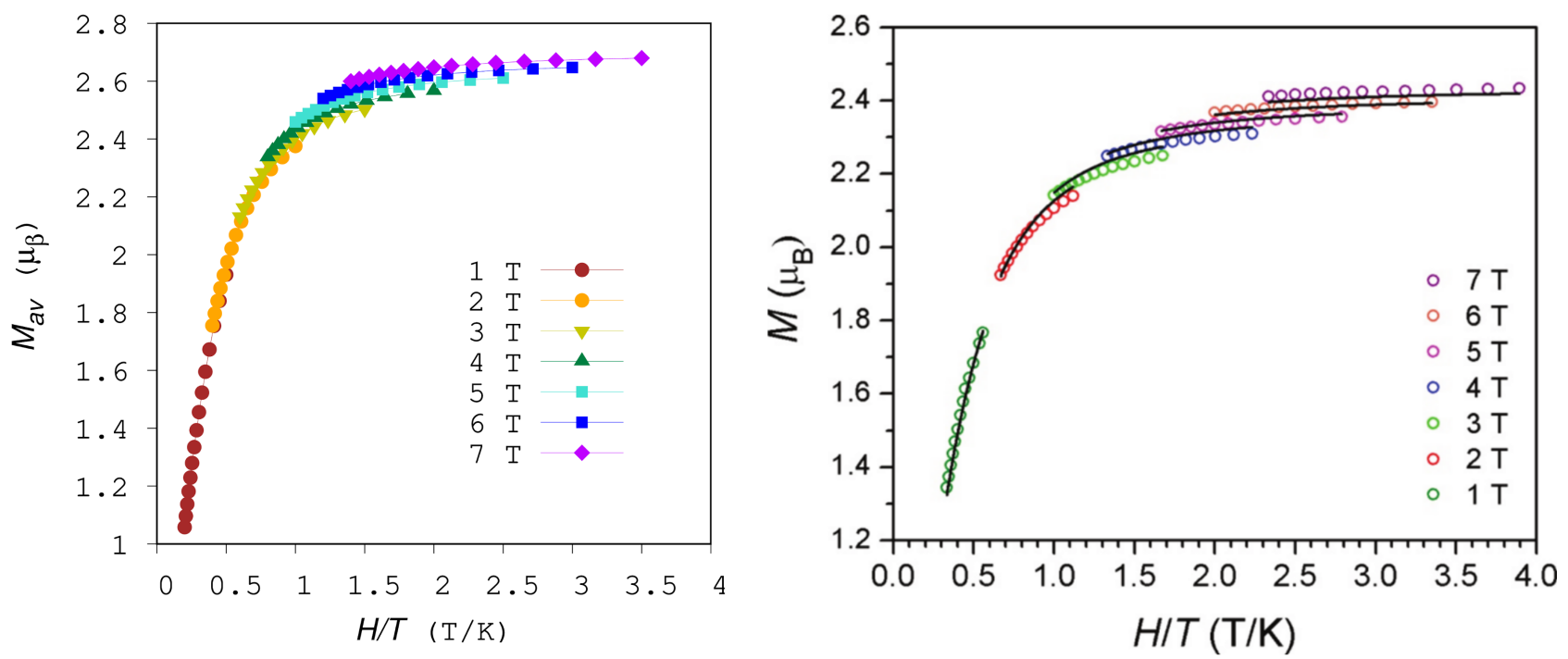

Figure S4: Left: Calculated isofield magnetization curves $(M$ versus $|\mathbf{H}| / T)$ of $[(\mathrm{tpa}) \mathrm{Fe}(\mathrm{II})]^{-}$. Magnetization is in Bohr magneton $\left(\mu_{b}\right)$ units. For each field $(|\mathbf{H}|$ from 1 to $7 \mathrm{~T})$ the temperature is increased from 2 to $5 \mathrm{~K}$. Right: Experimental isofield curves of [( $\left.\left.\mathrm{tpa}^{\mathrm{Tbu}}\right) \mathrm{Fe}\right]^{-}$from Ref. 2 .
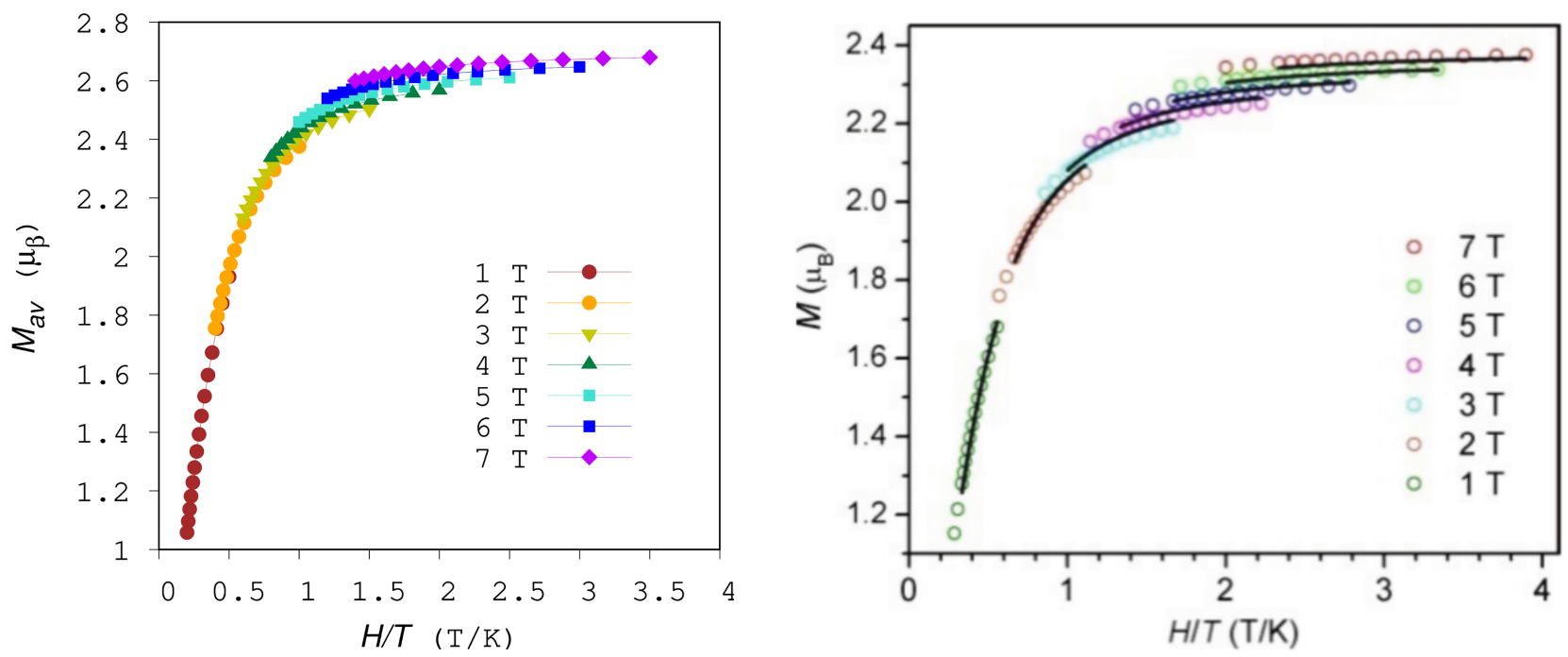

Figure S5: Left: Calculated isofield magnetization curves $(M$ versus $|\mathbf{H}| / T)$ of $[(\mathrm{tpa}) \mathrm{Fe}(\mathrm{II})]^{-}$. Magnetization is in Bohr magneton $\left(\mu_{b}\right)$ units. For each field $(|\mathbf{H}|$ from 1 to $7 \mathrm{~T})$ the temperature is increased from 2 to $5 \mathrm{~K}$. Right: Experimental isofield curves of $\left[\left(\mathrm{tpa}^{\mathrm{Mes}}\right) \mathrm{Fe}\right]^{-}$from Ref. 3. 

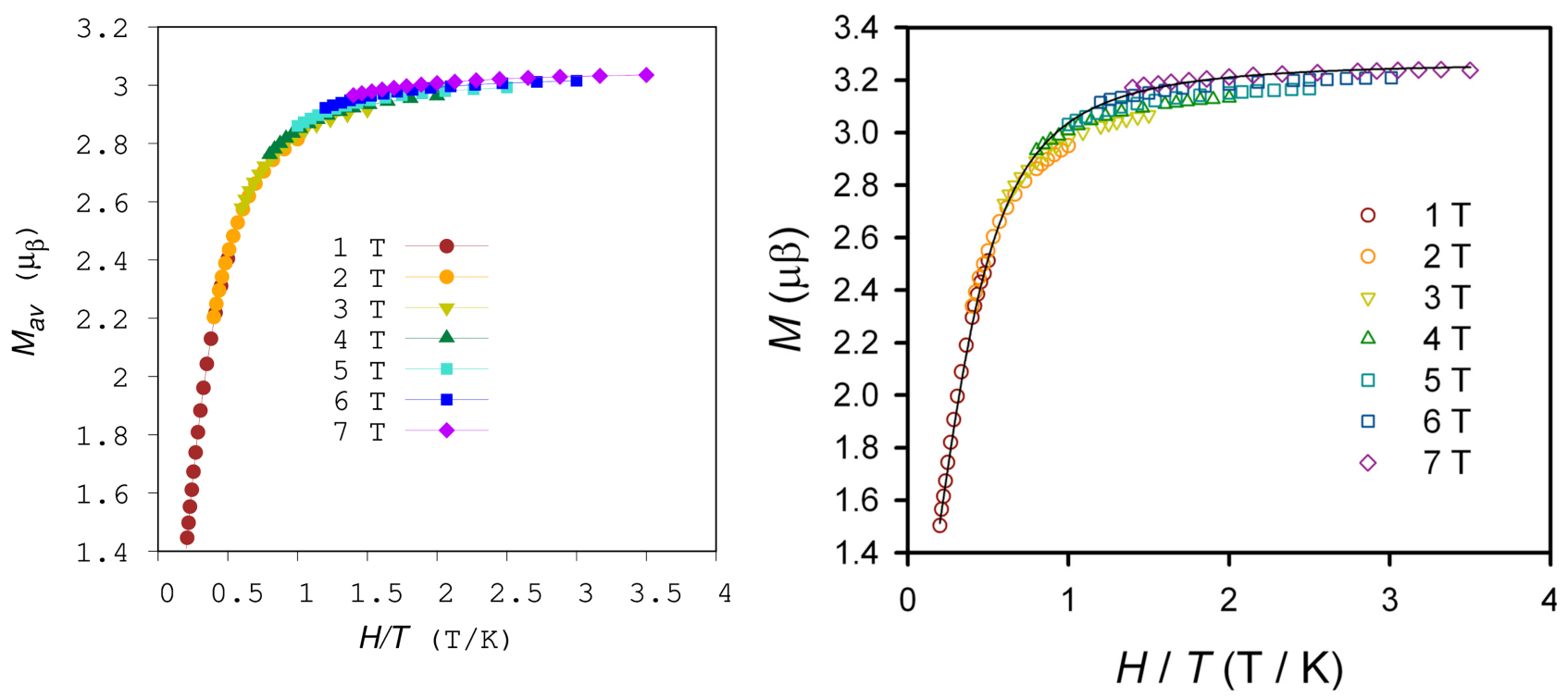

Figure S6: Left: Calculated isofield magnetization curves $(M$ versus $|\mathbf{H}| / T)$ of $\mathrm{Fe}(\mathrm{II})\left[\mathrm{C}\left(\mathrm{SiMe}_{3}\right)_{3}\right]_{2}$. Magnetization is in Bohr magneton $\left(\mu_{b}\right)$ units. For each field $(|\mathbf{H}|$ from 1 to $7 \mathrm{~T}$ ) the temperature is increased from 2 to $5 \mathrm{~K}$. Right: Experimental isofield curves of $\mathrm{Fe}(\mathrm{II})\left[\mathrm{C}\left(\mathrm{SiMe}_{3}\right)_{3}\right]_{2}$ from Ref. 4.
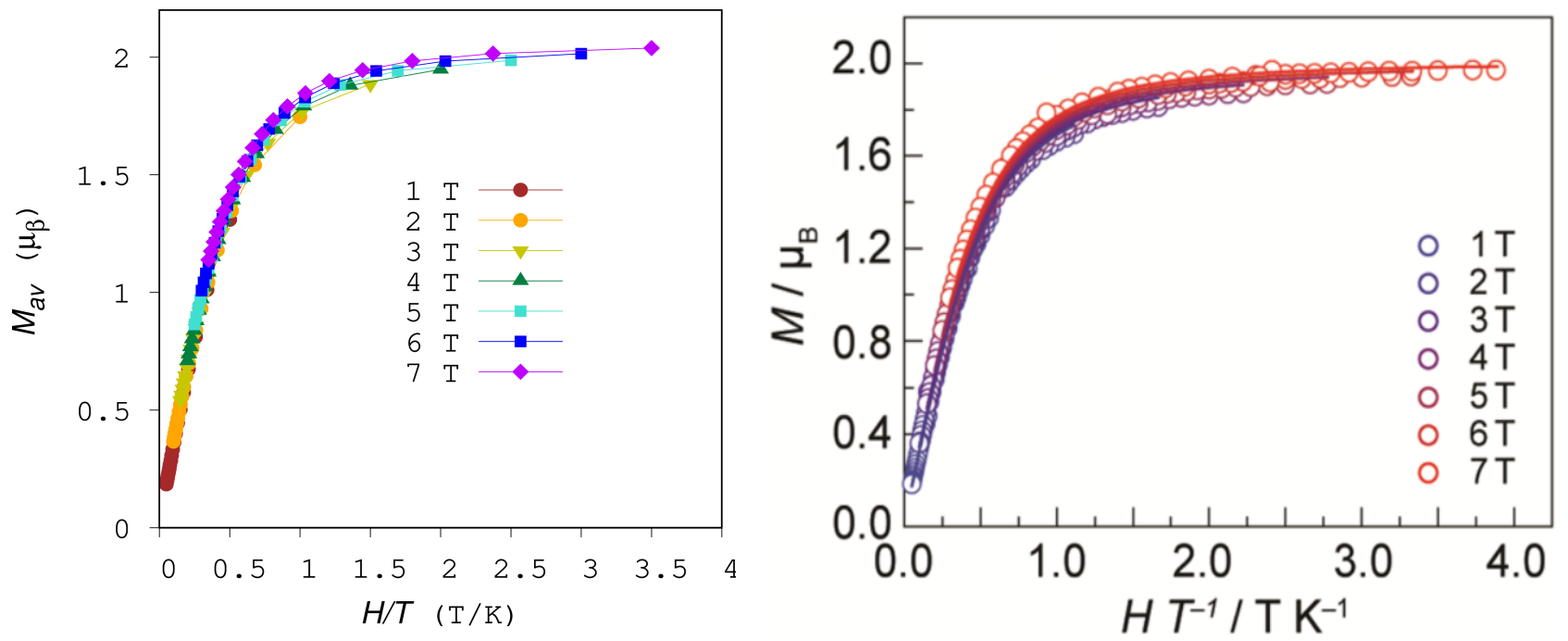

Figure S7: Left: Calculated isofield magnetization curves $(M$ versus $|\mathbf{H}| / T)$ of $\left(\mathrm{PMe}_{3}\right)_{2} \mathrm{Fe}(\mathrm{III}) \mathrm{Cl}_{3}$. Magnetization is in Bohr magneton $\left(\mu_{b}\right)$ units. For each field $(|\mathbf{H}|$ from 1 to $7 \mathrm{~T}$ ) the temperature is increased from 2 to $20 \mathrm{~K}$. Right: Experimental isofield curves of $\left(\mathrm{PMe}_{3}\right)_{2} \mathrm{Fe}(\mathrm{III}) \mathrm{Cl}_{3}$ from Ref. 5 . 


\section{Convergence of magnetization and susceptibility data with the number of states}

The Boltzmann distribution gives the probability that a system is in a certain state as a function of the state's energy and temperature of the system:

$$
p_{i}=\frac{e^{\frac{-E_{i}}{k T}}}{\sum_{n}^{N} e^{-\frac{E_{n}}{k T}}},
$$

where $p_{i}$ is the probability (population) of state $i, E_{i}$ is the energy of state $i$ and $N$ is the number of states accessible to the system. Table S4 reports the Boltzmann distribution of the doubly degenerate ground state and the first excited state for Fe(II) and Fe(III) compounds.

Table S4: Population analysis of the doubly degenerate ground state and the first excited state of the trigonal pyramidal $[(\mathrm{tpa}) \mathrm{Fe}(\mathrm{II})]^{-}$, linear $\mathrm{Fe}(\mathrm{II})\left[\mathrm{C}\left(\mathrm{SiMe}_{3}\right)_{3}\right]_{2}$, and trigonal bipyramidal $\left(\mathrm{PMe}_{3}\right)_{2} \mathrm{Fe}(\mathrm{III}) \mathrm{Cl}_{3}$ complexes. Energies are in $\mathrm{cm}^{-1}$.

\begin{tabular}{|c|c|c|c|c|c|}
\hline Fe(II) - Trigonal pyramidal & $E$ & $5 \mathrm{~K}$ & $50 \mathrm{~K}$ & $150 \mathrm{~K}$ & $300 \mathrm{~K}$ \\
\hline$|\mathrm{SOC}-1\rangle$ & -173 & 50 & 44 & 26 & 19 \\
\hline$|\mathrm{SOC}-2\rangle$ & -173 & 50 & 44 & 26 & 19 \\
\hline$|\mathrm{SOC}-3\rangle$ & -95 & 0 & 5 & 13 & 13 \\
\hline$|\mathrm{SOC}-4\rangle$ & -95 & 0 & 5 & 13 & 13 \\
\hline$|\mathrm{SOC}-5\rangle$ & -14 & 0 & 1 & 6 & 9 \\
\hline$|\mathrm{SOC}-6\rangle$ & -14 & 0 & 1 & 6 & 9 \\
\hline$|\mathrm{SOC}-7\rangle$ & 59 & 0 & 0 & 3 & 6 \\
\hline$|\mathrm{SOC}-8\rangle$ & 86 & 0 & 0 & 3 & 5 \\
\hline$|\mathrm{SOC}-9\rangle$ & 164 & 0 & 0 & 1 & 4 \\
\hline$|\mathrm{SOC}-10\rangle$ & 164 & 0 & 0 & 1 & 4 \\
\hline$|\mathrm{SOC}-11\rangle$ & 3719 & 0 & 0 & 0 & 0 \\
\hline$|\mathrm{SOC}-12\rangle$ & 3719 & 0 & 0 & 0 & 0 \\
\hline$|\mathrm{SOC}-13\rangle$ & 3733 & 0 & 0 & 0 & 0 \\
\hline$|\mathrm{SOC}-14\rangle$ & 3733 & 0 & 0 & 0 & 0 \\
\hline$|\mathrm{SOC}-15\rangle$ & 3738 & 0 & 0 & 0 & 0 \\
\hline
\end{tabular}




\begin{tabular}{|c|c|c|c|c|c|}
\hline $\mathrm{Fe}(\mathrm{II})$ - Linear & $E$ & $5 \mathrm{~K}$ & $50 \mathrm{~K}$ & $150 \mathrm{~K}$ & $300 \mathrm{~K}$ \\
\hline$|\mathrm{SOC}-1\rangle$ & -262 & 50 & 49 & 39 & 28 \\
\hline$|\mathrm{SOC}-2\rangle$ & -262 & 50 & 49 & 39 & 28 \\
\hline$|\mathrm{SOC}-3\rangle$ & -101 & 0 & 1 & 9 & 13 \\
\hline$|\mathrm{SOC}-4\rangle$ & -101 & 0 & 1 & 9 & 13 \\
\hline$|\mathrm{SOC}-5\rangle$ & 0 & 0 & 0 & 4 & 8 \\
\hline$|\mathrm{SOC}-6\rangle$ & 195 & 0 & 0 & 1 & 3 \\
\hline$|\mathrm{SOC}-7\rangle$ & 296 & 0 & 0 & 0 & 2 \\
\hline$|\mathrm{SOC}-8\rangle$ & 296 & 0 & 0 & 0 & 2 \\
\hline$|\mathrm{SOC}-9\rangle$ & 456 & 0 & 0 & 0 & 1 \\
\hline$|\mathrm{SOC}-10\rangle$ & 456 & 0 & 0 & 0 & 1 \\
\hline$|\mathrm{SOC}-11\rangle$ & 1690 & 0 & 0 & 0 & 0 \\
\hline$|\mathrm{SOC}-12\rangle$ & 1690 & 0 & 0 & 0 & 0 \\
\hline$|\mathrm{SOC}-13\rangle$ & 1691 & 0 & 0 & 0 & 0 \\
\hline$|\mathrm{SOC}-14\rangle$ & 1691 & 0 & 0 & 0 & 0 \\
\hline$|\mathrm{SOC}-15\rangle$ & 1592 & 0 & 0 & 0 & 0 \\
\hline Fe(III) - Trigonal bipyramidal & $E$ & $5 \mathrm{~K}$ & $50 \mathrm{~K}$ & $150 \mathrm{~K}$ & $300 \mathrm{~K}$ \\
\hline$|\mathrm{SOC}-1\rangle$ & -198 & 50 & 49 & 36 & 26 \\
\hline$|\mathrm{SOC}-2\rangle$ & -198 & 50 & 49 & 36 & 26 \\
\hline$|\mathrm{SOC}-3\rangle$ & -69 & 0 & 1 & 11 & 14 \\
\hline$|\mathrm{SOC}-4\rangle$ & -69 & 0 & 1 & 11 & 14 \\
\hline$|\mathrm{SOC}-5\rangle$ & 91 & 0 & 0 & 3 & 6 \\
\hline$|\mathrm{SOC}-6\rangle$ & 91 & 0 & 0 & 3 & 6 \\
\hline$|\mathrm{SOC}-7\rangle$ & 213 & 0 & 0 & 1 & 4 \\
\hline$|\mathrm{SOC}-8\rangle$ & 213 & 0 & 0 & 1 & 4 \\
\hline$|\mathrm{SOC}-9\rangle$ & 2166 & 0 & 0 & 0 & 0 \\
\hline$|\mathrm{SOC}-10\rangle$ & 2106 & 0 & 0 & 0 & 0 \\
\hline$|\mathrm{SOC}-11\rangle$ & 2119 & 0 & 0 & 0 & 0 \\
\hline$|\mathrm{SOC}-12\rangle$ & 2119 & 0 & 0 & 0 & 0 \\
\hline$|\mathrm{SOC}-13\rangle$ & 2144 & 0 & 0 & 0 & 0 \\
\hline$|\mathrm{SOC}-14\rangle$ & 2144 & 0 & 0 & 0 & 0 \\
\hline
\end{tabular}



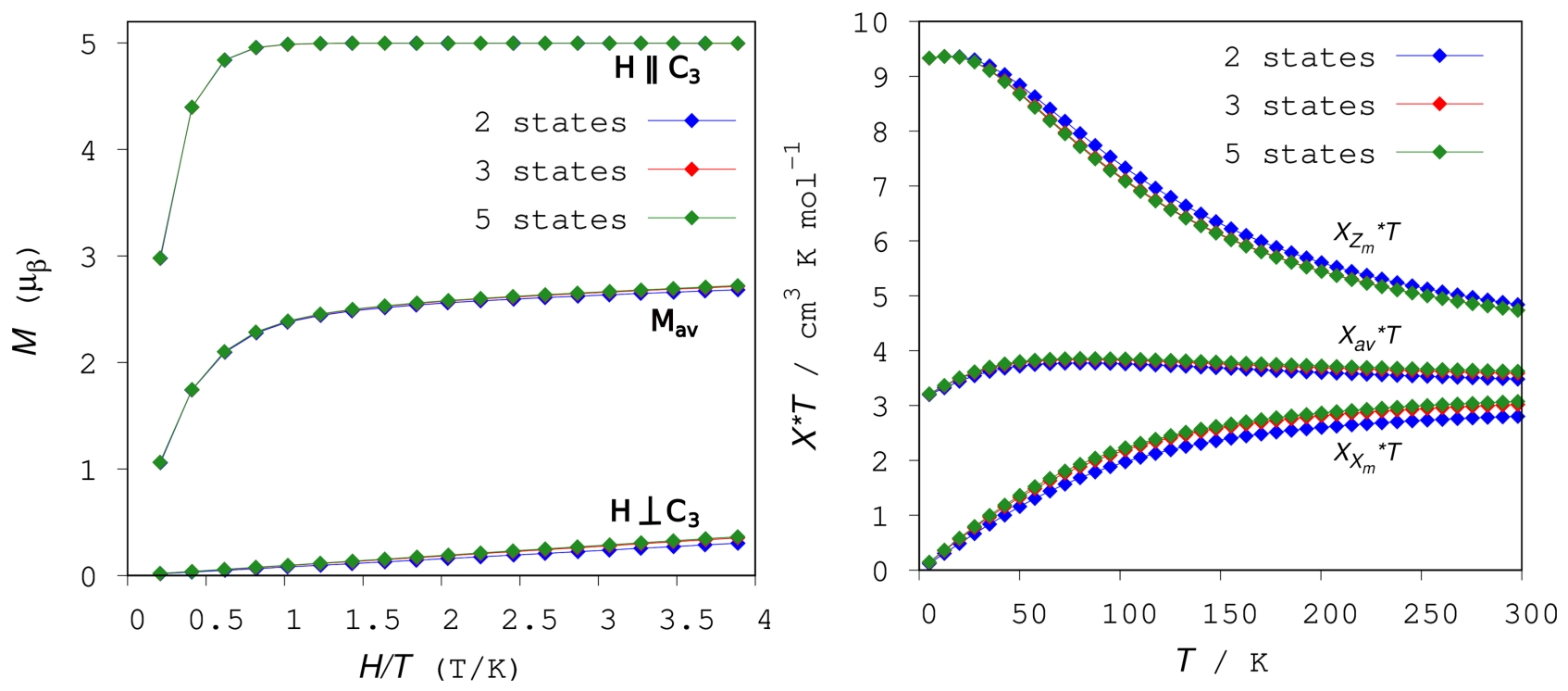

Figure S8: Calculated magnetization (left) and susceptibility (right) plots of [(tpa)Fe(II)] $]^{-}$. Results are derived using 2 (blue curves), 3 (red curves), and 5 (green curves) states.
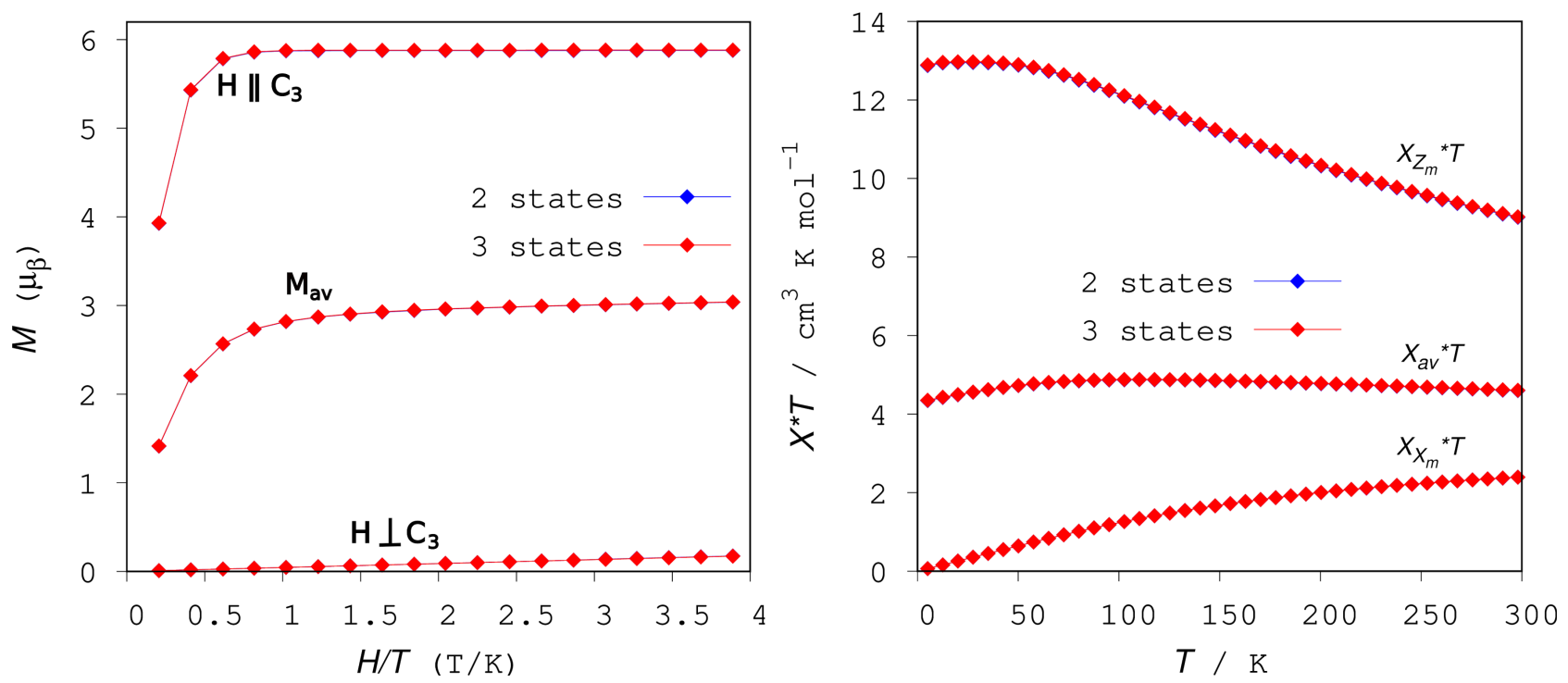

Figure S9: Calculated magnetization (left) and susceptibility (right) plots of $\mathrm{Fe}(\mathrm{II})\left[\mathrm{C}\left(\mathrm{SiMe}_{3}\right)_{3}\right]_{2}$. Results are derived using 2 (blue curves) and 3 (red curves) states. 

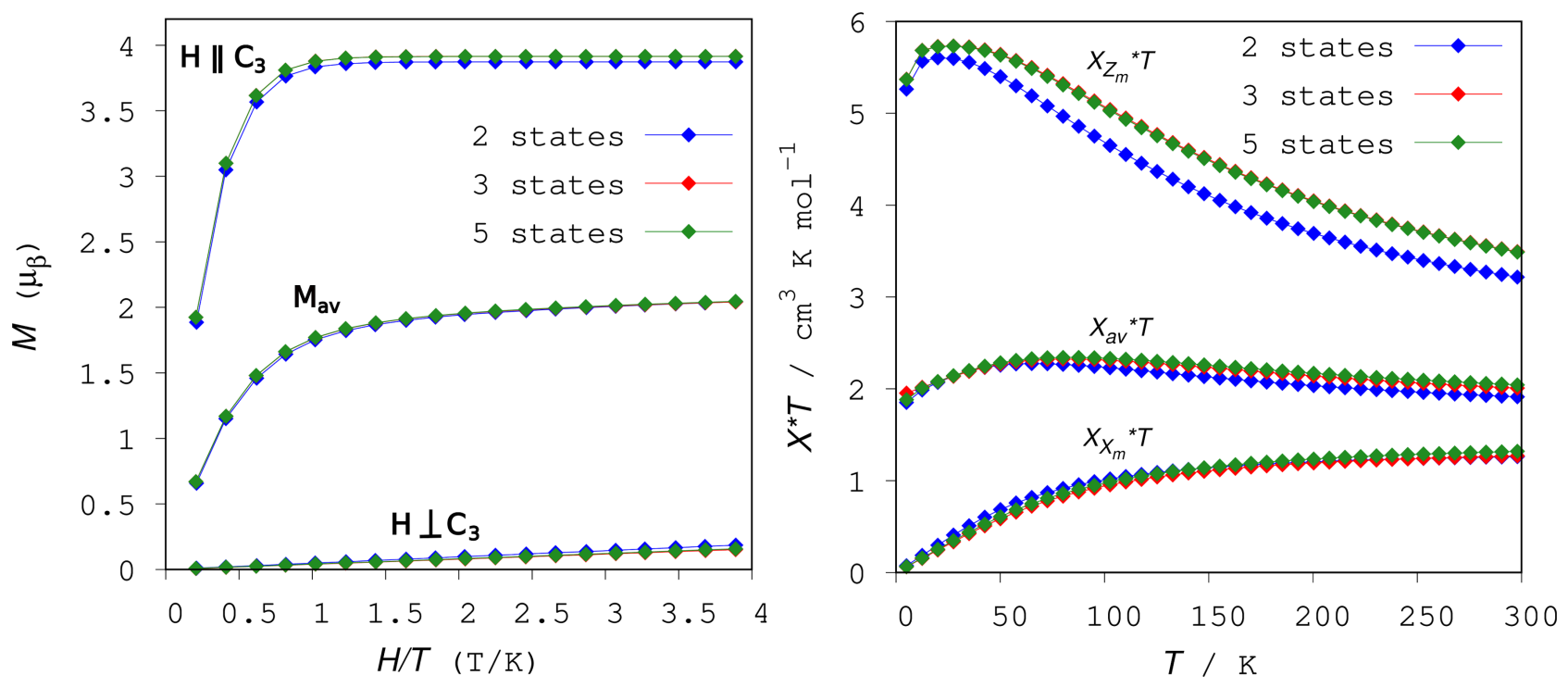

Figure S10: Calculated magnetization (left) and susceptibility (right) plots of $\left(\mathrm{PMe}_{3}\right)_{2} \mathrm{Fe}(\mathrm{III}) \mathrm{Cl}_{3}$. Results are derived using 2 (blue curves), 3 (red curves), and 5 (green curves) states. 


\section{$7 \quad$ Basis-set effects}

Table S5: Energy barrier $U$ (in $\mathrm{cm}^{-1}$ ) of $\left(\mathrm{PMe}_{3}\right)_{2} \mathrm{Fe}(\mathrm{III}) \mathrm{Cl}_{3}$ computed for the doubly degenerate ground state and with the cc-pVDZ and cc-pVTZ basis sets.

\begin{tabular}{lcc}
\hline Basis set & $U$ & $U_{\text {eff }}$ \\
\hline cc-pVDZ & 103 & \\
cc-pVTZ & 89 & 81 \\
Exp. ${ }^{a, b}$ & 100 & 8 \\
\hline
\end{tabular}

${ }^{a}$ Energy barrier $\left(U=D\left(S^{2}-1 / 4\right)\right)$ from experimental $D$ parameter and $S$ spin. Effective energy barriers $\left(U_{\text {eff }}\right)$ from ac susceptibility measurements. ${ }^{b}$ Ref. 5.
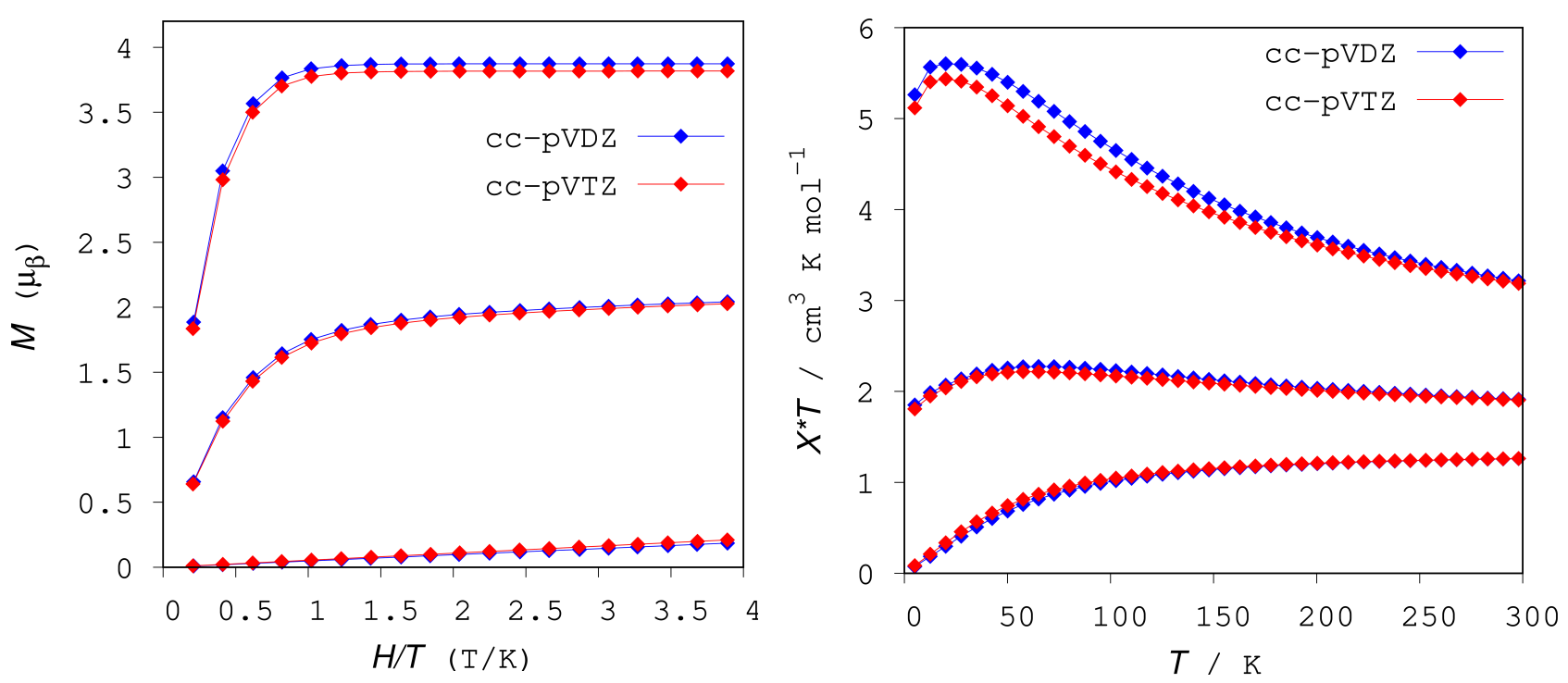

Figure S11: Calculated magnetization (left) and susceptibility (right) plots of $\left(\mathrm{PMe}_{3}\right)_{2} \mathrm{Fe}(\mathrm{III}) \mathrm{Cl}_{3}$ obtained with the cc-pVDZ (blue curves) and cc-pVTZ (red curves) basis sets. 


\section{References}

[1] Pokhilko, P.; Krylov, A. I. Quantitative El-Sayed rules for many-body wavefunctions from spinless transition density matrices J. Phys. Chem. Lett. 2019, 10, 4857-4862.

[2] Harman, W. H.; Harris, T. D.; Freedman, D. E.; Fong, H.; Chang, A.; Rinehart, J. D.; Ozarowski, A.; Sougrati, M. T.; Grandjean, F.; Long, G. J.; Long, J. R.; Chang, C. J. Slow magnetic relaxation in a family of trigonal pyramidal iron(II) pyrrolide complexes J. Am. Chem. Soc. 2010, 132, 18115-18126.

[3] Freedman, D. E.; Harman, W. H.; Harris, T. D.; Long, G. J.; Chang, C. J.; Long, J. R. Slow magnetic relaxation in a high-spin iron(II) complex J. Am. Chem. Soc. 2010, 132, 1224-1225.

[4] Zadrozny, J. M.; Atanasov, M.; Bryan, A. M.; Lin, C.; Rekken, B. D.; Power, P. P.; Neese, F.; Long, J. R. Slow magnetization dynamics in a series of two-coordinate iron(II) complexes Chem. Sci. 2013, 4, 125-138.

[5] Feng, X.; Hwang, S. J.; Liu, J.-L.; Chen, Y.-C.; Tong, M.-L.; Nocera, D. G. Slow magnetic relaxation in intermediate spin $\mathrm{S}=3 / 2$ mononuclear Fe(III) complexes J. Am. Chem. Soc. 2017, 139, 16474-16477.

[6] Head-Gordon, M. Characterizing unpaired electrons from the one-particle density matrix Chem. Phys. Lett. 2003, 372, 508-511. 


\title{
Relevant Cartesian coordinates
}

\author{
\$comment \\ Neutral hextet (tpa)Fe structure \\ Optimized with wB97XD/cc-pVDZ (J. Chem. Phys. 151, 034106, 2019) \\ Nuclear Repulsion Energy $=1977.63586011$ hartrees \\ \$end \\ \$molecule \\ 06 \\ N $-0.0009594129-0.0005151132$ \\ 1.2715236551 \\ N $1.3796505581-1.3423679276$ \\ $-0.6796869350$ \\ N -1.8531795328 \\ $-0.5112691392$ \\ $-0.6807247131$ \\ N $\quad 0.4744716301$ \\ 1.8639100138 \\ $-0.6803344605$ \\ C 1.1451037230 \\ 2.8312819863 \\ $-1.3939265612$ \\ C 0.4217802546 \\ 2. 2980622639 \\ 0.6398265889 \\ C 1.4983579229 \\ 3. 8656841193 \\ $-0.5571924750$ \\ C 1.0421397333 \\ 3. 5194454229 \\ 0.7510412251 \\ C -2.2030430284 \\ $-0.7797532699$ \\ 0.6381174299 \\ C -3.0256606760 \\ $-0.4189921773$ \\ $-1.3959756544$ \\ C -4.0980133907 \\ $-0.6367821660$ \\ $-0.5607584307$ \\ C -3.5707117890 \\ $-0.8596599045$ \\ 0.7474974740 \\ C 1.8857694100 \\ $-2.4045491730$ \\ $-1.3941612512$ \\ C 1.7795278098 \\ $-1.5151271547$ \\ 0.6411864849 \\ C 2.5285507710 \\ $-2.6622603345$ \\ 0.7519965822 \\ C 2.6041637627 \\ $-3.2278802178$ \\ $-0.5569987154$ \\ C 1.3583354911 \\ $-0.4748939165$ \\ 1. 6338671040 \\ C -0.2702946696 \\ 1. 4142135906 \\ 1. 6321594602 \\ C -1.0921896812 \\ $-0.9409254395$ \\ 1.6303855035 \\ H 2.0299585686 \\ 4.7692029943 \\ $-0.8454370880$ \\ H 1.1504775701 \\ 4.1046208613 \\ 1.6613974936 \\ H -5.1464335392 \\ $-0.6320435527$ \\ $-0.8492763773$ \\ $\mathrm{H}-4.1337641022$ \\ $-1.0619170971$ \\ 1. 6558315805 \\ H 2.9776577184 \\ $-3.0528505066$ \\ 1.6623583170 \\ H 3.1241574763 \\ $-4.1380127924$ \\ $-0.8456881405$ \\ H 2.0146733390 \\ 0.4089366821 \\ 1.5930025488 \\ H 1.3701401594 \\ $-0.8641303596$ \\ 2. 6652410494 \\ 1. 5421160095 \\ 1. 5900163807 \\ 1. 6195866609 \\ 2.6642430144 \\ H 0.0583583554 \\ $-1.9512839337$ \\ 1. 5841045064 \\ H -0.6550743392 \\ $-0.7609728844$ \\ 2. 6629427641 \\ H 1.3294318038 \\ 2.7092430480 \\ $-2.4588421637$ \\ $\mathrm{H}-3.0130140952$ \\ $-0.1972049798$ \\ $-2.4606855314$ \\ H 1.6918753419 \\ $-2.5031502086$ \\ $-2.4598148605$ \\ Fe 0.0009029075 \\ 0.0000323988 \\ $-0.8532015669$ \\ \$end
}


\$comment

Experimental structure of $\mathrm{Fe}(\mathrm{C}(\mathrm{SiMe3}) 3) 2$ (Z. Anorg. Allg. Chem. 627, 715, 2001) Nuclear Repulsion Energy $=5026.85808590$ hartrees \$end

\begin{tabular}{|c|c|c|c|}
\hline \multicolumn{4}{|c|}{ \$molecule } \\
\hline$F_{0}$ & -0.0000000051 & -0.0000003502 & 0.00 \\
\hline & 0.0174962578 & -0.0001 & 3871 \\
\hline & 1.6080485019 & -0.8701529216 & -2.496 \\
\hline & 3.1379191991 & 0.2151469177 & -2.380 \\
\hline & & -0.2 & -2 \\
\hline & 3.23677 & 0.53 & -1 \\
\hline & 3.045 & & -2 \\
\hline & 1.949 & -2 & -1 \\
\hline & 2.7691201805 & -2.7 & -1 \\
\hline & & -2 . & -1 \\
\hline & 2.026 & -1 & -0 . \\
\hline & & -1 & -4 \\
\hline & & -2 & -4 \\
\hline & 1.501 & $-0 . \varepsilon$ & -4 \\
\hline & & -2 . & -4 \\
\hline$i$ & & -0 . & -2 \\
\hline & -1.34 & -2 & -2 \\
\hline & -0.57 & -3 & -3 \\
\hline & -2.133 & -3.2 & -2 \\
\hline & -1.26 & -3 & -1 \\
\hline & & -0 . & -4 \\
\hline & -1.10 & -0 . & -5 \\
\hline & -2.10 & 0.3 & 739 \\
\hline & -2.611 & -1.1 & -4 \\
\hline & -3.020 & -0 & -1 \\
\hline & -2.862 & -0.6 & -0.7 \\
\hline & -3.749 & -1 & -1 \\
\hline & -3.242 & 0.3 & -1 \\
\hline$\perp$ & -0.000 & & -2 . \\
\hline & 0.634 & 2. & -4.3 \\
\hline T & $0.11^{\circ}$ & & -4 \\
\hline & 1.55 & 701 & -4 \\
\hline & 0.556079 & 2.9 & -4.5 \\
\hline & -1.676 & 2.6 & -2 . \\
\hline & -2.2754116093 & 2.1344 & -3.1804255559 \\
\hline & -1.5904738678 & & -2.9290887711 \\
\hline & -2.0264137025 & 2.6319827438 & -1.7140026436 \\
\hline $\mathrm{C}$ & 1.0269948399 & 2.8536779487 & -1.4678362214 \\
\hline & 1.916090317 & 2.4934596200 & 1.412477 \\
\hline
\end{tabular}




\begin{tabular}{|c|c|c|c|}
\hline $\mathrm{H}$ & 0.6289108530 & 604355471 & -0 \\
\hline $\mathrm{H}$ & 1.0660319914 & 3.7494662122 & -1.8096552860 \\
\hline & -0.0174962680 & 0.0001 & 2.04528 \\
\hline & -1.6080485121 & 0.8701522212 & 2.4965374059 \\
\hline & -3.1379192093 & -0.2151476181 & 2.3808432695 \\
\hline & -3.9111656372 & 0.2965319556 & 2.6274194406 \\
\hline & -3.2367702583 & -0.5344273274 & 1.4815093325 \\
\hline & -3.0459469582 & -0.9615232906 & 2.9786 \\
\hline & -1.9496508434 & 2.2808598063 & 1.3123235101 \\
\hline & -2.7691163923 & 1783912 & 1.5587501318 \\
\hline & -1.2280768227 & 2.9129039294 & 1.3503723398 \\
\hline & -2.0264856662 & 2419432 & 0.418 \\
\hline & -1.6265950246 & 1.58 & 4.23 \\
\hline & -2.4701435698 & 2.0118206761 & 4.3952041237 \\
\hline & -1.5014448249 & 0853677 & 4.8727 \\
\hline & -0.918 & 2.2256662395 & 4.325 \\
\hline $\mathrm{Si}$ & 1.485 & 414 & 2.669 \\
\hline & 1.3 & 2.8 & 2.53 \\
\hline & 0.5724598496 & 3.1137206673 & 3.0112580441 \\
\hline & 2.1334630505 & 6869 & 388040 \\
\hline & 762009 & 1212283 & 231490 \\
\hline & 043715 & 742 & 4.469 \\
\hline & 1.105 & 0.7 & 5.006 \\
\hline & 2.104 & -0.3282477964 & 4.5733433322 \\
\hline $\mathrm{H}$ & 2.611 & 3574 & 4.747 \\
\hline & 3.020 & 0.5 & 1.693 \\
\hline & 2.862 & 7787 & 193897 \\
\hline & 3.749 & $1.0^{\circ}$ & 1.99 \\
\hline & 790586 & 4455480 & 1.8194341892 \\
\hline $\mathrm{Si}$ & 024618 & -1.7 & 250651 \\
\hline $\mathrm{C}$ & -0.634247 & -2.0 & 914310 \\
\hline & -0.117 & -1.4 & 460035 \\
\hline $\mathrm{H}$ & -1.556590 & -1.767739 & 600094 \\
\hline & -0.5560791979 & -2.9583242718 & 4.5984800254 \\
\hline & 1.676273 & -2.6214998943 & 2.6082535621 \\
\hline $\mathrm{H}$ & 2.2754115991 & -2.1344267061 & 3.1804257142 \\
\hline & 1.5904738576 & -3.5220684287 & 2.9290889294 \\
\hline & 2.0264136923 & -2.6319834443 & 1.7140028019 \\
\hline C & -1.0269885378 & -2.8536710929 & 1.4678346309 \\
\hline & -1.9160928411 & -2.4934604604 & 1.4124684078 \\
\hline & -0.6289108632 & -2.8604362476 & 0.5942777136 \\
\hline & -1.0660320015 & -3.7494669127 & 1.8096554443 \\
\hline
\end{tabular}


\$comment

Experimental structure of (PMe3)2Fe(III)Cl3 (J. Am. Chem. Soc. 139, 16474, 2017) Nuclear Repulsion Energy $=1941.14025377$ hartrees

\$end

\$molecule

06

C

$-1.5927571560$

$-3.1487581758$

$-1.4826190042$

$-4.1177034744$

$\mathrm{H} \quad-0.7444591908$

$-2.1269133300$

$-2.8007341653$

$\mathrm{H} \quad 0.8461332535$

$-2.0492899006$

$-2.9585062251$

C $\quad 1.4586498138$

0.9008675183

$-3.0116432036$

1.8224122881

$-2.6773969001$

1.4745492340

0.9093148005

$-3.9908374895$

0.4350076011

$-2.7242287062$

$-1.6013900361$

3. 0764157229

$-1.5224608648$

4.0459273615

$-2.1320832157$

2.6955163181

$-2.0428220257$

2.8913646693

0.8879027743

3.0143510247

0.4817662976

2.6451640861

1.8385964716

2.7776799310

0.7949618143

3. 9896846766

0.0420022507

$-2.3490068875$

0.0515371925

2.3301814969

2. 2264308598

0.0121073906

$-1.2406202111$

0.0218882312

$-0.0225847387$

$-0.0024111887$

0.9005273894

$-3.0116706276$

1. 8220684262

$-2.6774246251$

0.9089846932

$-3.9908641055$

0.4344780523

$-2.7242714028$

0.8882430012

3.0143784566

0.4822958464

2. 6452067827

1.8389302630

2. 7777068440

0.7953027290

3. 9897121640

$-1.2401741976$

0.0219241925

\$end 